\title{
Prioritizing systemic therapies for genitourinary malignancies: Canadian recommendations during the COVID-19 pandemic
}

\author{
Aly-Khan A. Lalani, BSc(Hons), MD ${ }^{1}$; Kim N. Chi, MD²; Daniel Y.C. Heng, MD, \\ $\mathrm{MPH}^{3}$; Christian K. Kollmannsberger, $\mathrm{MD}^{2}$; Srikala S. Sridhar, MD, MSc${ }^{4}$; Normand \\ Blais, MD, MSc ${ }^{5}$; Christina Canil, MD ${ }^{6}$; Piotr Czaykowski, MD, MSc ${ }^{7}$; Sebastien J. \\ Hotte, MD, $\mathrm{MSc}^{1}$; Nayyer Iqbal, $\mathrm{MD}^{8}$; Denis Soulières, MD, $\mathrm{MSc}^{5}$; Dominick Bossé, \\ MD, MSc, MPH ${ }^{6}$; Nimira S. Alimohamed, MD³ Naveen S. Basappa, MD ${ }^{11}$; Som D. \\ Mukherjee, $\mathrm{MD}^{1}$; Eric Winquist, $\mathrm{MD}, \mathrm{MSc}^{9}$; Lori A. Wood, $\mathrm{MD}^{10}$; Scott A. North, MD, \\ MHPE $^{11}$ \\ ${ }^{1}$ Juravinski Cancer Centre, McMaster University, Hamilton, ON, Canada; ${ }^{2} \mathrm{BC}$ Cancer Agency, Vancouver \\ Cancer Center, Vancouver, BC, Canada; ${ }^{3}$ Tom Baker Cancer Centre, University of Calgary, Calgary, AB, \\ Canada; ${ }^{4}$ Princess Margaret Cancer Centre, University of Toronto, Toronto, ON, Canada; ${ }^{5}$ Centre \\ Hospitalier de l'Université de Montréal, Montreal, QC, Canada; ${ }^{6}$ The Ottawa Hospital Cancer Centre, \\ University of Ottawa, Ottawa, ON, Canada; ${ }^{7}$ Cancer Care Manitoba, University of Manitoba, Winnipeg, \\ MB, Canada; ${ }^{8}$ Saskatoon Cancer Centre, University of Saskatchewan, Saskatoon, SK, Canada; ${ }^{9}$ London \\ Health Sciences Centre, Western University, London, ON, Canada; ${ }^{10}$ Queen Elizabeth II Health Sciences \\ Centre, Dalhousie University, Halifax, NS, Canada; ${ }^{11}$ Cross Cancer Institute, University of Alberta, \\ Edmonton AB, Canada
}

Cite as: Can Urol Assoc J 2020 April 5; Epub ahead of print. http://dx.doi.org/10.5489/cuaj.6595

Published online April 5, 2020 


\section{Introduction}

The global outbreak of the novel coronavirus disease (COVID-19) is characterized by rapid human-to-human transmission of the severe adult respiratory syndrome coronavirus 2 (SARS-CoV-2) from droplet contamination. ${ }^{1}$ Initial reports from epicenters of the pandemic suggest that patients with a diagnosis of cancer may harbor a higher risk of SARS-CoV-2 infection. ${ }^{2}$ Data also highlights that hospital admission and recurrent hospital visits are risk factors for SARS-CoV-2 infection, and that receipt of anti-cancer therapy within 14 days of presentation is associated with higher rates of intensive care admission, mechanical ventilation, or death. ${ }^{3-5}$ Coupled with the significant constraints at upstream healthcare systems levels, it is incumbent upon oncologists to adopt prioritization strategies in order to deliver safe, effective, and feasible care in the setting of a pandemic. We sought to develop recommendations to assist in prioritizing systemic therapies for patients with genitourinary cancers in Canada.

\section{Guiding principles}

In situations of severely limited capacity - such as pandemics - ethical principles (justice, beneficence, non-maleficence, and autonomy) emphasize treating patients fairly, equitably, and safely, according to need. ${ }^{6}$ In the context of COVID-19, the value of "maximizing benefits" reflects the importance of responsible stewardship of resources. ${ }^{7}$ This framework is crucial when we consider how to triage cancer care in this climate. In general, highest priority is usually afforded in scenarios where a condition is immediately life-threatening or has a potential for high morbidity, or where definitive curative therapy has a significant chance of success. Higher range of priority is also applied to neo/adjuvant indications and non-curative situations with a significant prolongation of life (for example, $>1$ year of added life extension). Furthermore, considerations around best environments to assess patients, modalities for administration of comparable treatments, and supportive measures to help prevent hospital admission, are all valuable opportunities to optimize care. For example, use of G-CSF support can be considered on a case-by-case basis to determine if it may reduce the risk of febrile neutropenia and hospital presentation in an environment of escalating COVID-19 exposure. Ultimately, strategies should balance the acuity of patient need, standard of care guidelines, risk/benefit profiles of interventions, and significant resource constraints.

\section{Recommendations}

Eighteen academic genitourinary medical oncologists from 11 cancer centers across Canada participated in preparing this guidance document for managing patients during the current pandemic. The authors emphasize the need for frequent re-evaluation of prioritization strategies accounting for the trajectory of COVID-19, public health 
Prioritizing systemic therapies for GU malignancies during COVID-19

recommendations, and resources available at institutions in real time. The authors also recognize the importance of clear discussion and documentation of prognosis, performance status, and patients' goals of care in medical records since these are also pivotal inputs in triaging COVID-19 related healthcare resources for these patients, should they be required.

\section{Prostate cancer}

Scenario: Treatment for metastatic hormone-sensitive disease (mHSPC)

- Recommendation for androgen-receptor-axis targeted therapies (ARATs), in preference over docetaxel chemotherapy, as treatment intensification in addition to androgen deprivation therapy (ADT).

- While docetaxel also has evidence for improved overall survival in mHSPC, chemotherapy carries more frequent resource utilization and risks of toxicities (particularly neutropenia) in an escalating COVID-19 pandemic.

- The initiation of ARATs can be delayed up to 6 months post initiation of ADT in this climate.

Scenario: First-line therapy for metastatic castration-resistant disease (mCRPC)

- Recommendation for ARATs, when these therapies have not been used previously.

- If ARATs have been used previously (in the nonmetastatic-CRPC or mHSPC settings) such that chemotherapy would be next-line recommendation, patient and physician discussion should reflect on: (1) whether chemotherapy initiation can be safely delayed; (2) whether patient comorbidities place them at higher risk of COVID-19; (3) potential hospital constraints at that time to manage intravenous administration and chemotherapy-related adverse events.

- In patients with bone-only mCRPC, radium-223 may be a preferred option over chemotherapy, assuming availability.

Scenario: Second-line therapy for metastatic castration-resistant disease (mCRPC)

- Recommendation for ARATs, when these therapies have not been used previously.

- If ARATs have been used prior such that chemotherapy would be next-line recommendation, patient and physician discussion should reflect on: (1) whether chemotherapy initiation can be safely delayed; (2) whether patient comorbidities place them at higher risk of COVID-19; (3) potential hospital constraints at that time to manage intravenous administration and chemotherapy-related adverse events. 
- In patients with bone-only mCRPC, radium-223 may be a preferred option over chemotherapy, assuming availability.

Scenario: Third-line therapy for metastatic castration-resistant disease (mCRPC)

- Recommendation for alternate ARATs over alternate chemotherapy, in patients suitable for further treatment.

- While level I data supports an alternate chemotherapy for some patients in this setting, the magnitude of benefit in the current climate is balanced by hospital constraints to manage intravenous administration and chemotherapy-related adverse events.

- In patients with bone-only mCRPC, radium-223 may be a preferred option over chemotherapy, assuming availability.

Scenario: Glucocorticoids as part of anticancer therapy for prostate cancer

- Glucocorticoids should be minimized as an adjunct to systemic therapies in an escalating COVID-19 pandemic. If indicated, the lowest effective dose to achieve the therapeutic outcome should be used.

\section{Urothelial carcinoma}

Scenario: Neoadjuvant cisplatin-based combination chemotherapy (NAC) for muscleinvasive disease

- Multi-disciplinary conversations between uro-oncology, radiation oncology, and medical oncology are critical, particularly to assess resource availability in order to deliver standard of care in the current context.

- In situations where access to definitive surgery is available: the benefits of NAC may be outweighed by associated risks and resource utilization. Surgical and supportive post-operative care resources may be limited in the future if there are large numbers of COVID-19 patients requiring admission, which may jeopardize timely access to subsequent radical cystectomy. Adjuvant chemotherapy can be considered and, where indicated, potentially delayed post-operatively.

- In situations where access to definitive surgery is unavailable: initial NAC provides a curative intent systemic treatment. However, the risks associated with NAC must be carefully weighed in a patient population particularly vulnerable to COVID-19 and in context of available resources to manage toxicities.

- Definitive radiation to the bladder should be particularly considered, especially as surgical availability and healthcare resources attributed to NAC are constantly evolving. The shortest course of effective radiation should be planned to limit 
hospital visits. Concurrent radio-sensitizing monotherapy with weekly cisplatin or gemcitabine are preferred, if resources are available.

- If NAC is deemed appropriate, we recommend a minimum of 3 cycles in the current climate to limit toxicities and promptly move onto definitive therapy. We recommend not using dose-dense regimens in this climate.

Scenario: First-line therapy for advanced urothelial carcinoma (aUC)

- Recommendation for platinum-based doublet chemotherapy up to a maximum of 6 cycles. The marginal benefit beyond 4 cycles in this setting warrants serious consideration for treatment duration.

- In select patients with a low burden of disease, delaying the start of systemic therapy could be considered in an escalating pandemic. Local therapy (i.e. radiation) to the primary and/or oligo-metastatic sites may assist in this regard but should be discussed with multi-disciplinary input.

Scenario: Therapy for platinum-refractory aUC

- Recommendation is for checkpoint inhibitor therapy (CPI) in eligible patients.

- For patients currently benefitting from CPI, physician and patient discussion should reflect on whether treatment can be held. Where safe and available, longer interval dosing of CPI should be employed.

- Chemotherapy should be carefully considered in an escalating COVID-19 pandemic and may be attempted in select, eligible patients with disease that warrants urgent treatment.

\section{Renal cell carcinoma}

Scenario: First line therapy for IMDC good risk advanced renal cell carcinoma (aRCC)

- If systemic therapy is not acutely indicated: active surveillance is recommended.

- If systemic therapy is required: oral VEGF-targeted therapy (VEGF-TT) is recommended.

- When considering the addition of intravenous CPI to VEGF-TT, patient and physician discussion should reflect on: (1) resource utilization for intravenous treatment; (2) potential hospital constraints to manage immune-related adverse events (irAEs); and (3) the incremental benefit of CPI in this subgroup.

- For patients currently benefitting from first-line therapy (i.e. $>6$ months), physician and patient discussion should reflect on whether treatment can be held. Where safe and available, longer interval dosing of CPI should be employed. 
Prioritizing systemic therapies for GU malignancies during COVID-19

Scenario: First line therapy for IMDC intermediate/poor risk aRCC

- Recommendation for combination therapy with CPI (dual CPI or CPI/VEGF-TT) in eligible patients. In the COVID-19 climate, CPI/VEGF-TT may be preferred over dual CPI due to less irAEs and potential for prolonged use of corticosteroids.

- Prior to initiation of new therapy, patient and physician discussion should reflect on: (1) whether treatment initiation can be delayed during an escalating COVID19 period; and (2) if hospital resource constraints will allow for both intravenous treatment and adequate management of high grade iRAEs.

- If the COVID-19 situation is particularly volatile, treatment initiation with VEGFTT may be a more reasonable option to minimize risk of severe iRAEs.

- For patients currently benefitting from first-line therapy (i.e. $>6$ months), physician and patient discussion should reflect on whether treatment can be held. Where safe and available, longer interval dosing of CPI should be employed.

Scenario: Second line therapy and beyond for aRCC

- Prior to new treatment initiation, considerations should balance resource utilization, and assessment and treatment intervals, for potential next line options.

- For patients currently benefitting from CPI monotherapy (i.e. $>6$ months), physician and patient discussion should reflect on whether treatment can be held. Where safe and available, longer interval dosing of CPI should be employed.

- In an escalating COVID-19 pandemic, careful consideration of risks and potential benefits should be given prior to commencing third-line and beyond therapies if disease progression has already been demonstrated on both VEGF-TT and CPI therapies.

Scenario: Timing of Cytoreductive Nephrectomy $(\mathrm{CN})$

- In general, upfront $\mathrm{CN}$ should be avoided or delayed in light of surgical restraints. In the event of a significant response to upfront systemic therapy, a $\mathrm{CN}$ could be considered after multi-disciplinary discussion and once hospital resources resume normal practice.

\section{Germ cell tumours (GCTs)}

Scenario: Adjuvant chemotherapy for stage I GCTs

- Recommendation not to initiate adjuvant chemotherapy in the current climate, on the basis of balancing the benefit profile with resource utilization and hospital constraints. 
- Surveillance programs are recommended utilizing virtual care pathways, outpatient laboratory tests, and imaging in local facilities to minimize hospital contact. Careful consideration should be given when scheduling laboratory tests and imaging, particularly in years 2-5 of surveillance.

- Patients with respiratory symptoms who are scheduled for regular GCT laboratory tests or imaging can be safely delayed for 1-2 weeks until resolution of symptoms or identification of cause.

Scenario: Treatment for Stage II seminoma

- Recommendation that these patients be discussed in a multi-disciplinary setting, since treatment may be safely delayed during an escalating COVID-19 pandemic (i.e. radiation therapy for stage IIA). At a later time, chemotherapy may be a feasible option.

Scenario: Treatment for IGCCCG good-risk disseminated GCT

- Recommendation for BEP x 3 cycles, unless contraindication to bleomycin, given manageable short and long-term toxicities compared to EP x 4 cycles.

- The need for PFT's should be judiciously assessed since this testing is associated with a higher risk of infection transmission and access to PFT's may be restricted.

- In good risk patients with a concurrent COVID-19 diagnosis, preference is to consider safely delaying chemotherapy initiation for viral clearance. If treatment cannot be delayed, BEP x 3, EP x 4 and VIP x 3 are reasonable options.

- In patients with concurrent COVID-19 induced pneumonia and need for immediate treatment, EP x 4 and VIP $\times 3$ are preferred options over bleomycin containing regimens.

- Delays in surgical management should be kept to a minimum if possible. Outpatient procedures (diagnostic biopsies or excision) should not be delayed if possible.

- Whenever possible, modifications to the standard management plan such as delaying chemotherapy or post-chemotherapy surgery patients should be discussed with an expert center.

Scenario: Treatment for IGCCCG intermediate or poor-risk disseminated GCT

- Recommendation for VIP x 4 or BEP x 4. Due to higher risk of bleomycininduced pulmonary toxicity with 4 cycles (compared to 3), VIP x 4 may be preferred in the COVID-19 pandemic depending on regional hospital resources. 
- Certain patients with intermediate risk disease (by blood markers only without anatomic bulk disease) and concurrent significant viral illness may be closely observed to allow symptom resolution and viral clearance.

- It is not an option to delay treatment in patients with poor risk disease.

- The need for PFT's should be judiciously assessed since this testing is associated with a higher risk of infection transmission and access to PFT's may be restricted.

- In these patients with a concurrent COVID-19 diagnosis, recommendation is for VIP $\mathrm{x} 4$ assuming safe to do so.

- Delays in surgical management should be kept to a minimum if possible. Outpatient procedures (diagnostic biopsies or excision) should not be delayed if possible.

- These patients should all be discussed with an expert center.

Scenario: Salvage chemotherapy for relapsing disease

- Recommendation that these patients be discussed urgently with an expert center to formulate an appropriate care plan in the current context of COVID-19.

\section{Virtual care}

- Institutional logistical support should be provided for telephone and video conferencing modalities to facilitate patient assessment, tumour board discussion and, where suitable, treatment.

- Disease site teams should assess which new consultations can be safely seen in virtual setting (i.e. stage I GCTs entering active surveillance programs) versus face-to-face encounters (i.e. multi-disciplinary clinics for muscle-invasive urothelial carcinoma). Follow up encounters should be per physician discretion.

- Wherever possible, laboratory and imaging tests should be facilitated locally for patients to avoid travel and tertiary hospital settings, which may be COVID-19 treatment facilities. Depending on the clinical scenario, some imaging tests may be safely delayed beyond standard intervals in an escalating pandemic.

- Health care infrastructure should be nimble to ensure a coordinated approach when delivering virtual care for patients, including outpatient pharmacy mechanisms to allow the ordering of anti-cancer or supportive therapy outside of usual office/clinic settings (i.e. phone and encrypted electronic ordering) and for delivery of oral treatment to patient's homes.

- For the necessary in-person patient encounters, institutions should ensure safe physical distancing among health-care providers in clinics. This may also require 
physician teams rotating between on-site and off-site schedules to limit their exposure.

\section{Conclusions}

These recommendations, framed within a Canadian context, provide guidance for genitourinary oncology care during the COVID-19 pandemic and are intended to be reassessed as the situation requires. In this regard, the recommendations may also be informative in future pandemics or situations of dire resource restrictions. Studies that evaluate durable learning lessons from COVID-19, with respect to how and in what form we have adapted our practices, are forthcoming. 


\section{References}

1. Guan WJ, Ni ZY, Hu Y, et al. Clinical Characteristics of Coronavirus Disease 2019 in China. The New England journal of medicine. 2020.

2. Yu J, Ouyang W, Chua MLK, Xie C. SARS-CoV-2 Transmission in Patients With Cancer at a Tertiary Care Hospital in Wuhan, China. JAMA oncology. 2020.

3. Ng OT, Marimuthu K, Chia PY, et al. SARS-CoV-2 Infection among Travelers Returning from Wuhan, China. The New England journal of medicine. 2020.

4. Onder G, Rezza G, Brusaferro S. Case-Fatality Rate and Characteristics of Patients Dying in Relation to COVID-19 in Italy. Jama. 2020.

5. Zhang L, Zhu F, Xie L, et al. Clinical characteristics of COVID-19-infected cancer patients: A retrospective case study in three hospitals within Wuhan, China. Annals of Oncology (2020), doi: https://doi.org/10.1016/j.annonc.2020.03.296

6. Persad G, Wertheimer A, Emanuel EJ. Principles for allocation of scarce medical interventions. Lancet (London, England). 2009;373:423-31.

7. Emanuel EJ, Persad G, Upshur R, et al. Fair Allocation of Scarce Medical Resources in the Time of Covid-19. The New England journal of medicine. 2020. 


\section{Competing interests:}

Dr. Lalani has received honoraria from Astellas Pharma, Bayer, Bristol-Myers Squibb, Merck, Novartis, Pfizer, Roche/Genentech, and Tersera; has had a consulting or advisory role with Abbvie, Astellas Pharma, Bayer, Bristol-Myers Squibb, Eisai, Ipsen, Janssen, Merck, Pfizer, Roche/Genentech, and Tersera; and has received research funding from Bristol-Myers Squibb (Inst), Ipsen, Novartis, and Roche. Dr. Chi has received honoraria from Astellas Pharma, Bayer, Janssen, and Sanofi; has had a consulting or advisory role with Amgen, Astellas Pharma, AstraZeneca, Bayer, ESSA, Janssen, Roche, and Sanofi; and has received research funding from Astellas Pharma (Inst), Bayer (Inst), Bristol-Myers Squibb (Inst), Janssen (Inst), Lilly/ImClone (Inst), Merck (Inst), Roche (Inst), Sanofi (Inst), and Tokai Pharmaceuticals (Inst). Dr. Heng has had a consulting or advisory role with Astellas Pharma, Bristol-Myers Squibb, Eisai, Ipsen, Janssen, Merck, Novartis, and Pfizer; and has received research funding from Bristol-Myers Squibb (Inst), Exelixis (Inst), Ipsen (Inst), Novartis (Inst), and Pfizer (Inst). Dr. Kollmannsberger has received honoraria from Bristol-Myers Squibb, Novartis, and Pfizer; has had a consulting or advisory role with Astellas Pharma, Bristol-Myers Squibb, Eisai, Ipsen, Janssen, Novartis, and Pfizer; and has received travel, accommodation and expenses from Eisai, Novartis, and Pfizer. Dr. Sridhar has had a consulting or advisory role with Astellas Pharma (Inst), AstraZeneca (Inst), Bayer (Inst), Bristol-Myers Squibb (Inst), Janssen (Inst), Merck (Inst), Roche/Genentech (Inst), and Sanofi (Inst); and has received research funding from Bayer (Inst), Janssen (Inst), and Sanofi (Inst). Dr. Blais has had a consulting or advisory role with AstraZeneca, Bayer, Boehringer Ingelheim, Bristol-Myers Squibb, Merck, Pfizer, Roche Canada, and Sanofi Canada; and as received research funding from Merck. Dr. Canil has received honoraria from Janssen; has had a consulting or advisory role with AstraZeneca, Bayer, BristolMyers Squibb, Eisai, EMD Serono, Ipsen, Janssen, Merck, Pfizer, and Roche; has received research funding from Eisai; and has received travel, accommodations, and expenses from Amgen, Pfizer, Sanofi, and Janssen. Dr. Czaykowski has received research funding from Argos Therapeutics (Inst), Merck (Inst), Millennium (Inst), and Pfizer (Inst). Dr. Hotte has received honoraria from Astellas Scientific and Medical Affairs Inc, Bayer, Eisai Canada, and Janssen Oncology; has had a consulting or advisory role with Astellas Pharma; AstraZeneca, Bayer, Bristol-Myers Squibb, Eisai, Janssen, Merck, Pfizer, and Roche Canada; has received research funding from AstraZeneca, Ayala Pharmaceuticals, Bayer, Bristol-Myers Squibb, Clovis Oncology, Janssen Oncology, Merck, Pfizer (Inst), and Roche/Genentech; and has received travel, accommodations, and expenses from Eisai Canada. Dr. Iqbal has received honoraria from Janssen, Merck, Pfizer, and Novartis; has had a consulting or advisory role with Bristol-Myers Squibb, Merck, Pfizer, and Roche Canada; and has received travel, accommodations, and 
expenses from Novartis. Dr. Soulières has received honoraria from AstraZeneca, BristolMyers Squibb, Ipsen, Merck, Novartis, Pfizer, and Roche/Genentech; has had a consulting or advisory role with Ipsen, Merck, and Pfizer; and has received research funding from Bristol-Myers Squibb (Inst), Lilly (Inst), Merck (Inst), Novartis (Inst), Pfizer (Inst), and Roche/Genentech (Inst). Dr. Bossé has received honoraria from Amgen, AstraZeneca, Ipsen, and Janssen; has had a consulting or advisory role with Abbvie, Bayer, Bristol-Myers Squibb, Ipsen, and Pfizer; and has received research funding from AstraZeneca. Dr. Alimohamed has had a consulting or advisory role with Astellas Pharma, AstraZeneca, Janssen, Merck, Pfizer, and Sanofi Canada. Dr. Basappa has received honoraria fromo Astellas Pharma, Eisai, Ipsen, Janssen, Merck, and Pfizer; has had a consulting or advisory role with Astellas Pharma, AstraZeneca, Bristol-Myers Squibb, Eisai, Ipsen, Janssen, Merck, Pfizer, and Roche Canada; and has received travel, accommodations, and expenses from Eisai and Janssen. Dr. Mukherjee has received honoraria from AstraZeneca; has had a consulting or advisory role with Merck, Novartis/Pfizer, and Roche; and has received travel, accommodations, and expenses from Amgen. Dr. Winquist has received honoraria from Amgen, Bayer, Eisai, Merck, and Roche; and has received research funding from AstraZeneca/MedImmune (Inst), BristolMyers Squibb (Inst), Eisai (Inst), Merck (Inst), Pfizer (Inst); and Roche/Genentech (Inst). Dr. Wood has received research funding from Aragon Pharmaceuticals (Inst), AstraZeneca (Inst), Bristol-Myers Squibb (Inst), Exelixis (Inst), Merck (Inst), Novartis (Inst), Pfizer (Inst), and Roche Canada (Inst). Dr. North has received honoraria from Astellas Pharma, Janssen-Ortho, Merck, Novartis, Pfizer, Roche Canada, and Sanofi Canada; has had a consulting or advisory role with Astellas Pharma, AstraZeneca, Janssen Oncology, Merck, Novartis, Pfizer, Roche Canada, and Sanofi Canada; has received research funding from Astellas Pharma (Inst), AstraZeneca (Inst), Janssen (Inst), Novartis Canada Pharmaceuticals Inc (Inst), Roche Canada (Inst), and Sanofi Canada (Inst); and has received travel, accommodations, and expenses from AstraZeneca. 